\title{
New Preparation Method for Highly Electrically Conductive Poly(pyrrole) Composite Film
}

\author{
Tohru YoshIKAwA, ${ }^{*}$ Shigeru MaChIDA, Takaaki IKEGAMI, \\ Anuntasin TECHAGUMPUCH, ${ }^{* *}$ and Seizo MIYATA \\ Department of Material Systems Engineering, Tokyo University of \\ Agriculture and Technology, Tokyo, Japan \\ * Omron Tateisi Electronics Co., Shiga, Japan \\ ** Department of Physics, Chulalongkorn University, \\ Bangkok, Thailand
}

(Received July 5, 1989)

\begin{abstract}
Conducting poly(pyrrole) composite films were prepared by chemical polymerization in oxidant solutions by dipping poly(ethylene terephthalate) film (PET film) coated with poly(methyl methacrylate) (PMMA) and pyrrole monomers into a $\mathrm{FeCl}_{3}$ aqueous solution. The oxidation potential of this solution was controlled by adding a suitbable amount of $\mathrm{FeCl}_{2}$ to the solution before the reaction. By selecting appropriate polymerization conditions such as oxidation potential, temperature, time and $\mathrm{FeCl}_{3}$ concentration, highly conducting poly(pyrrole) composite films could be obtained. The SEM studies of the synthesized films indicated that polymerization conditions which lead to high reaction rates often yielded films of aggregated structures which have
\end{abstract} low conductivities.

KEY WORDS Conducting Polymer / Poly(pyrrole) / Thin Film Polymer / Chemical Synthesized Polymer / Morphology / Oxidation Potential /

Electrical Conductivity /

In preparing conducting poly(pyrrole) films, three methods were previously reported: electrochemical polymerization, chemical polymerization and chemical vapor deposition (CVD). In 1979, Diaz reported that a highly conducting poly(pyrrole) could be prepared by an electrochemical method. ${ }^{1}$ Since then, many papers have been published. ${ }^{2-5}$ Recently K. Yoshino et al. reported that the applied potential during polymerization has influence on the conductivity of poly(pyrrole). The maximum conductivity of $c a .500 \mathrm{~S} \mathrm{~cm}^{-1}$ was observed at about $0.6 \mathrm{~V}$ ( $v s . \mathrm{SCE}){ }^{4}$ On the other hand, poly(pyrrole) obtained by the chemical method has shown only low conductivity. ${ }^{6-13}$ However, our group found that highly conducting poly(pyrrole) could be obtained if the oxidation potential was controlled to the optimum value during chemical polymerization.
In a previous report, poly(pyrrole) having a conductivity more than $200 \mathrm{~S} \mathrm{~cm}^{-1}$ was prepared. ${ }^{14}$ When this procedure was applied to CVD, poly(pyrrole) having a conductivity more than $100 \mathrm{~S} \mathrm{~cm}^{-1}$ was obtained. ${ }^{15}$ In this report, we describe a new preparation method for highly conducting poly(pyrrole) composite films and discuss the relationship between the conductivity and morphology of these films.

\section{EXPERIMENTAL METHOD}

Figure 1 shows how to prepare the conducting film. A poly(ethylene terephthalate) film (PET; thickness $=100 \mu \mathrm{m}$, Hoechst Synthesis Co., Ltd.) was used as a substrate film. For example, we coated poly(methyl methacrylate) (PMMA) which was dissolved in solvent on the PET film. After drying, this coated PET 


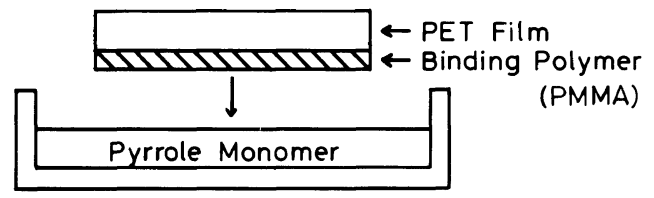

(a)

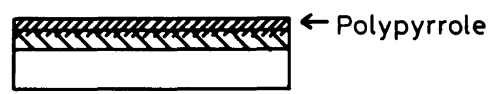

(c)

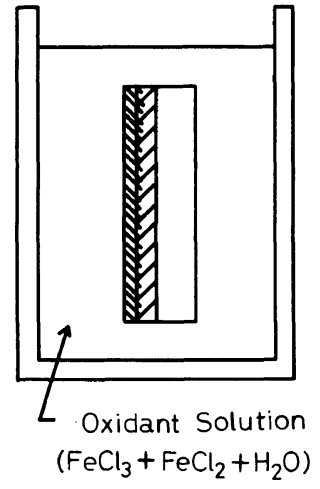

(b)

Figure 1. The preparation method for highly conductive poly(pyrrole) composite film.

film was dipped in purified pyrrole monomers for $5 \mathrm{~s}$ (Figure 1a). Then the film was transferred to a $\mathrm{FeCl}_{3}$ solution in distilled water whose oxidation potential was controlled (Figure $1 \mathrm{~b}$ ). Poly(pyrrole) was then synthesized in this $\mathrm{FeCl}_{3}$ solution.

It was observed that from Nernst's equation, ${ }^{16}$ the oxidation potential of $\mathrm{FeCl}_{3}$ solution in water,

$$
E=E_{0}+\frac{R T}{n F} \ln \frac{\left[\mathrm{FeCl}_{3}\right]}{\left[\mathrm{FeCl}_{2}\right]}
$$

depends on the molar ratio of $\mathrm{FeCl}_{3}$ to $\mathrm{FeCl}_{2}$ in the solution. Thus, by adding a small suitable amount of $\mathrm{FeCl}_{2}$ to the solution before the reaction, the oxidation potential can be controlled to a specific value as required. ${ }^{17}$

After preparation the film was dried under vacuum at $40^{\circ} \mathrm{C}$ for $24 \mathrm{~h}$. In measuring the conductivity of synthesized poly(pyrrole), the four-point probe method was used. First, four probes were applied to the surface of the poly(pyrrole) film still attached to the PMMA and PET films to measure the voltage/current ratio $(V / I)$ and obtained the surface resistance.

$$
R_{\mathrm{s}}=\frac{V \Pi}{I \ln 2}
$$

Later, the film was dipped into acetone to dissolve the PMMA, and the poly(pyrrole) film was separated from PET film. The thickness of the film $(d)$ was then measured using SEM. The conductivity of the poly(pyrrole) film was calculated by eq 3 .

$$
\sigma=\frac{1}{R_{\mathrm{s}} d}
$$

\section{RESULTS AND DISCUSSION}

Dependence of Conductivity on Oxidation Potential and $\mathrm{FeCl}_{3}$ Concentration

Figure 2 shows the change in conductivity of poly(pyrrole) films prepared with the initial oxidation potential ( $v s$. SCE) when $\mathrm{FeCl}_{3}$ concentrations were $1 \mathrm{moll}^{-1}$ and $3 \mathrm{moll}^{-1}$. In these preparations, soaking time in $\mathrm{FeCl}_{3}$ solution (polymerization time) was $5 \mathrm{~min}$ and soaking temperature (polymerization temperature) was $0^{\circ} \mathrm{C}$. The optimum oxidation potentials which yielded the highest conductivity values at each concentration were about $640 \mathrm{mV}$ and $600 \mathrm{mV}$ respectively. It is clear from this figure that $1 \mathrm{moll}^{-1} \mathrm{FeCl}_{3}$ solution yielded a poly(pyrrole) film of a conductivity as high as $110 \mathrm{~S} \mathrm{~cm}^{-1}$ which is much higher than that obtained from the $3 \mathrm{moll}^{-1} \mathrm{FeCl}_{3}$ solution. It was found that in the case of the 
$1 \mathrm{moll}^{-1} \mathrm{FeCl}_{3}$ solution, the polymerization of poly(pyrrole) did not occur or only partially so when the oxidation potential was lower than approximately $550 \mathrm{mV}$. To investigate the effect of $\mathrm{FeCl}_{3}$ concentration on the conductivity, we observed the morphology of the poly(pyrrole) films obtained from both solutions.

Figures 3(a) and 3(b) show surfaces of the poly(pyrrole) films obtained from $3 \mathrm{moll}^{-1}$ and $1 \mathrm{moll}^{-1}$ solutions, respectively. In the former case, the morphology of the film shows a large number of particles aggregated together, while the latter, the surface of the film

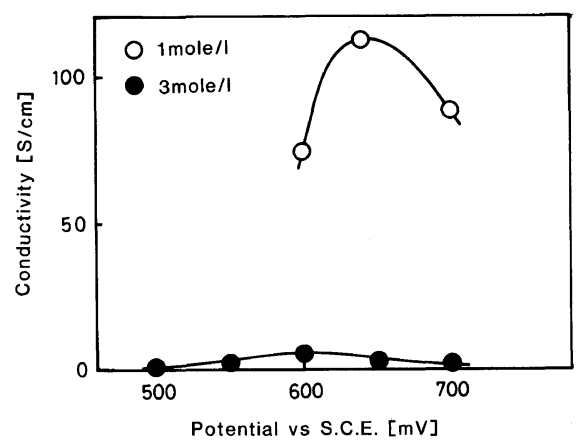

Figure 2. The conductivity of poly(pyrrole) film at various oxidation potentials when $\mathrm{FeCl}_{3}$ concentrations are $1 \mathrm{moll}^{-1}$ and $3 \mathrm{moll}^{-1}$. In this preparation, the soaking time (polymerization time) is $5 \mathrm{~min}$ and the soaking temperature (polymerization temperature) is $0{ }^{\circ} \mathrm{C}$.

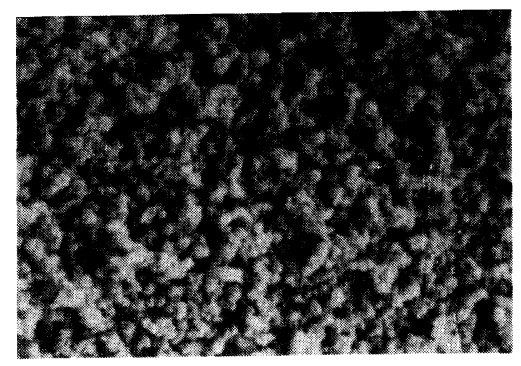

(a) is a smooth one with some particles on it. We consider that the aggregation structure in Figure 3(a) does not contribute to the conducting process while the smooth surface structure in Figure 3(b) does so. We propose that when the $\mathrm{FeCl}_{3}$ concentration was $3 \mathrm{moll}^{-1}$, the polymerization rate was too fast so that many polymerized nuclei evolved at the same time. This leads to an aggregated structure which results in decrease in conductivity.

\section{Dependence of Conductivity on Soaking Time}

Figure 4 shows the soaking time dependence of conductivity of poly(pyrrole) films prepared at $0 \mathrm{C}$ in $1 \mathrm{moll}^{-1} \mathrm{FeCl}_{3}$ solution of $640 \mathrm{mV}$ oxidation potential. The conductivity is maximum around $5 \mathrm{~min}$ soaking time. For a longer time, the conductivity drops steeply and finally levels off at about 7 to $8 \mathrm{~S} \mathrm{~cm}^{-1}$.

Figure 5 shows the changes in thickness and surface resistance of the poly(pyrrole) films in Figure 4 with soaking time. The surface resistance $\left(R_{\mathrm{s}}\right)$ drops down steeply at the beginning, but later is almost constant, while the thickness $(d)$ keeps on increasing with soaking time. According to eq 3 the conductivity of the film must decrease at a long soaking time as shown in Figure 4. This means that the conducting network is formed during the early time of polymerization, but after this the in-

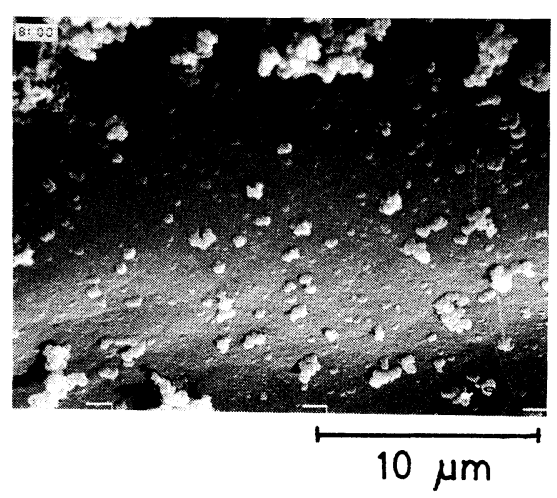

(b)

Figure 3. The morphology of prepared poly(pyrrole) films when $\mathrm{FeCl}_{3}$ concentration is (a) $3 \mathrm{moll}^{-1}$, or (b) $1 \mathrm{moll}^{-1}$. 
creasing part of the thickness seems not contribute to the conducting process. The SEM pictures of the front sides and backsides of

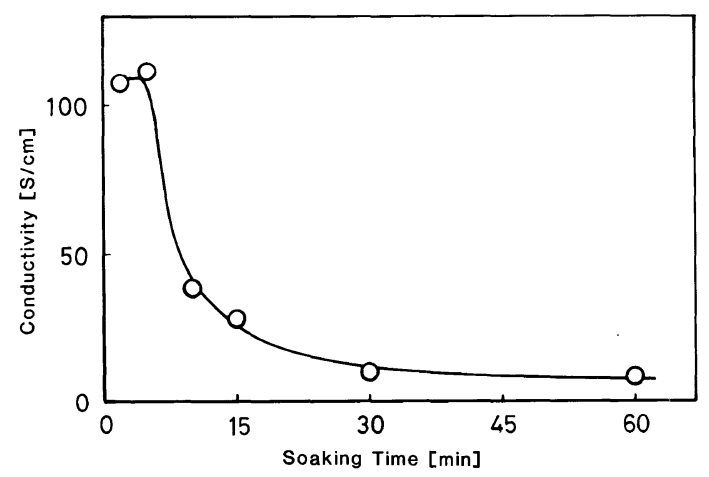

Figure 4. The conductivity of poly(pyrrole) films prepared in $1 \mathrm{moll}^{-1} \mathrm{FeCl}_{3}$ solution at various soaking times. The initial oxidation potential is $640 \mathrm{mV}$ and the soaking temperature is $0^{\circ} \mathrm{C}$.

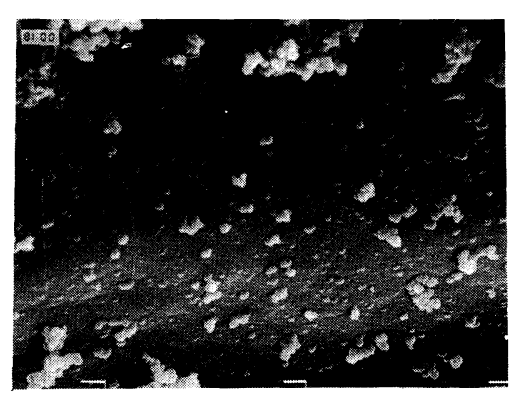

(a)

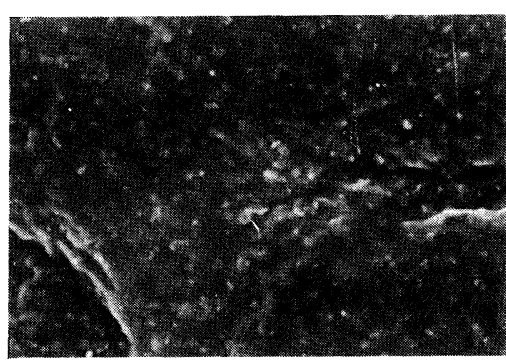

(c) these films also confirm this hypothesis.

Figures 6(a) and 6(b) show the front surface pictures of the films when the soaking times are $5 \mathrm{~min}$ and $1 \mathrm{~h}$, respectively. The morphology of these front surfaces is rather sim-

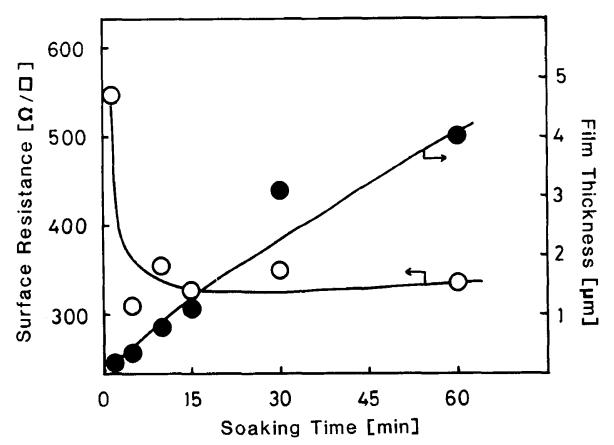

Figure 5. The surface resistance and thickness of prepared poly(pyrrole) films at various soaking times.

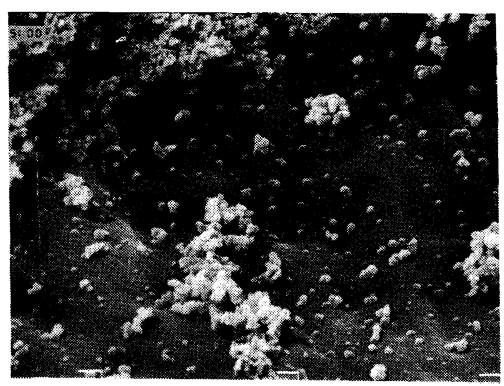

(b)

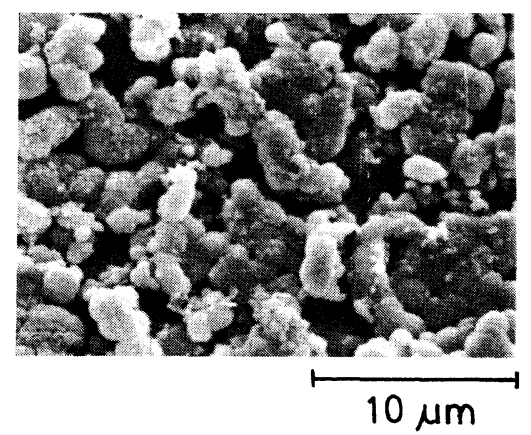

(d)

Figure 6. SEM pictures of poly(pyrrole) films. (a) front side, 5 min soaking time; (b) front side, $1 \mathrm{~h}$ soaking time; (c) backside, 5 min soaking time; (d) backside, 1 h soaking time. 
ilar. To investigate this problem further, the SEM pictures of the backsides of test films are shown in Figures 6(c) and 6(d) for $5 \mathrm{~min}$ and $1 \mathrm{~h}$ soaking, respectively.

In Figure 6(c), only small size particles are seen on the smooth surface. But in Figure 6(d), the number of particles increases and becomes an aggregated structure. It is clear that the thickness of this aggregated structure increases with polymerization time and this structure does not contribute to the conducting process of the poly(pyrrole) film. Since this film adhered very well with PMMA, cannot be separated from PMMA without dissolving the PMMA, we propose the origin of the aggregated structure as follows: When the PMMA film was dipped in pyrrole, some of the monomers dissolved in PMMA and formed a compatible binary structure. However as the polymerization proceeds, the phase separation took place and finally the synthesized poly(pyrrole) was separated from PMMA in order to minimize its surface energy and became the aggregated structure as seen in Figure 6(d). This picture also indicated that the poly(pyrrole) not only covered the surface of PMMA film but also penetrated inside the film to form a composite, resulting in strong adhesion between poly(pyrrole) and PMMA films.

\section{Dependence of Conductivity of Soaking Tem- perature}

Figure 7 shows soaking temperature dependence of conductivity. By increasing the temperature above $0 . \mathrm{C}$, the conductivity drops from $110 \mathrm{~S} \mathrm{~cm}^{-1}$ to $5 \mathrm{~S} \mathrm{~cm}^{-1}$ at $20^{\circ} \mathrm{C}$. This behavior can be explained by the morphology of the film prepared at $20^{\circ} \mathrm{C}$ shown in Figure 8 . In this picture, the aggregation of particles is observed more clearly than that obtained at $0 \mathrm{C}$ as shown in Figure 6(a). This structure can be explained as follows. At $20^{\circ} \mathrm{C}$, the reaction rate was very fast such that a large number of polymerized nuclei were evolved at the same time. This produced the structure of aggrega-

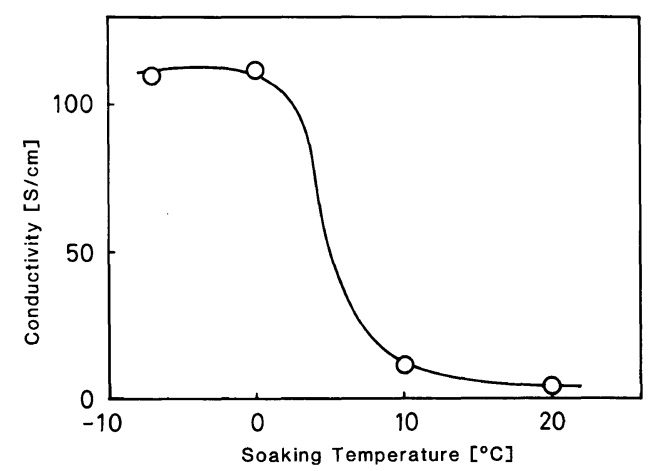

Figure 7. The conductivity of poly(pyrrole) films at various soaking temperatures. The soaking time is $5 \mathrm{~min}$ and $\mathrm{FeCl}_{3}$ concentration is $1 \mathrm{moll}^{-1}$.

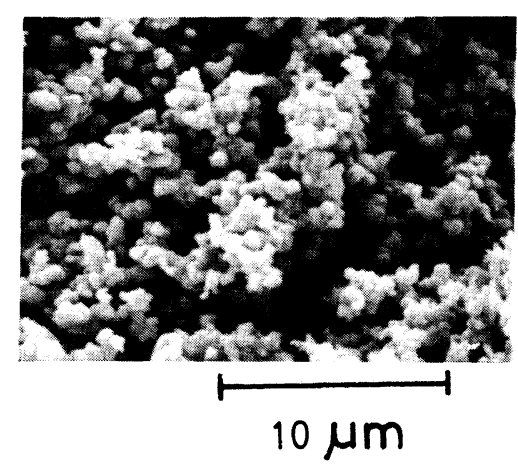

Figure 8. The morphology of the poly(pyrrole) film prepared at $20^{\circ} \mathrm{C}$.

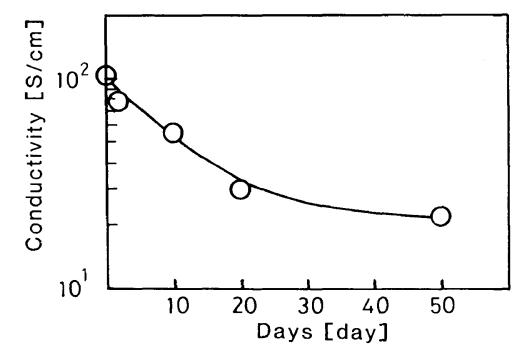

Figure 9. The stability of poly(pyrrole) films prepared in this work.

tion of many particles which reduced the conductivity of the film.

\section{Stability}

The stability of chemically synthesized poly(pyrrole) films is shown in Figure 9. The 
conductivity of these films decreased from $110 \mathrm{~S} \mathrm{~cm}^{-1}$ to $22 \mathrm{~S} \mathrm{~cm}^{-1}$ within 50 days and then rather leveled off.

\section{CONCLUSION}

A highly electrically conducting poly(pyrrole) composite film can be prepared by chemical polymerization in $\mathrm{FeCl}_{2}$ solution in water. It has been found that the following conditions, oxidation potential of $640 \mathrm{mV}, \mathrm{FeCl}_{3}$ concentration of about $1 \mathrm{moll}^{-1}$, soaking time of $5 \mathrm{~min}$, soaking temperature of $0^{\circ} \mathrm{C}$, are optimum reaction conditions for preparing poly(pyrrole) composite films of high conductivity. We also found that the morphology of prepared poly(pyrrole) films has profound influence on the conductivity. The films of aggregated structure seem to have low conductivity. Since the conductivity of the synthesized polypyrrole is rather stable at about $20 \mathrm{Scm}^{-1}$, we expect that these composite films can be used in electromagnetic shielding, circuit patterning and other similar applications.

Acknowledgments. This work was partially supported by a Grant-in-Aid for Scientific Research on Priority Areas, New Functionality Materials-Design, Preparation and Control (No. 63604007) from the Ministry of Education, Science, and Culture of Japan.

\section{REFERENCES}

1. A. F. Diaz, K. K. Kanazawa, and G. P. Gardini, $J$. Chem. Soc. Chem. Commun., 635 (1979).

2. G. B. Street, "Hand Book for Conducting Polymer," T. A. Skotheim, Ed., Marcel Dekker, Inc., New York and Basel, 1986, Chapter 8 and references therein.

3. S. J. Hahn, W. E. Stanchina, W. J. Gajda, and P. Vogelhut, J. Electron. Mater., 15, 145 (1986).

4. K. Yoshino, M. Tabata, M. Satoh, K. Kaneto, and T. Osawa, Tech. Rep. Osaka Univ., 35, 231 (1985).

5. M. Ogasawara, K. Funahashi, T. Hagiwara, and K. Iwata, Synth. Met., 14, 61 (1986).

6. A. Angeli, Gazz. Chim. Ital., 46, II, 279 (1916).

7. M. Salmon, K. Kanazawa, A. F. Diaz, and M. Krounbi, J. Polym. Sci. Polym. Lett. Ed., 22, 187 (1982).

8. M. Aizawa, H. Shinohara, and H. Shirakawa, Polym. Prepr. Jpn., 33, 495 (1984).

9. G. Bidan, CEA Report R, 5321 (1985).

10. T. Iyoda, A. Ohtani, T. Kaneko, M. Ando, M. Aiba, T. Shimidzu, and K. Honda, Polym. Prepr. Jpn., 35, 2682 (1986).

11. T. Shimidzu, T. Iyoda, and K. Fukui, Kasen Kouensyu, 43, 51 (1986).

12. T. Ojio and S. Miyata, Polym. J., 18, 95 (1986).

13. A. Mohammadi, I. Lundstrom, W. R. Salaneck, and O. Inganas, Synth. Met., 21, 169 (1987).

14. S. Machida and S. Miyata, Polym. Prepr. Jpn., 36, 1886 (1987).

15. T. Ikegami, S. Machida, S. Miyata, and T. Yoshikawa, Polym. Prepr. Jpn., 37, 738 (1988).

16. A. J. Bard and L. R. Faulkner, "Electrochemical Methods," John Wileys and Sons, New York, N.Y., 1980, p 51.

17. S. Machida, A. Techagumpuch, and S. Miyata, Synth. Met., 31, 311 (1989). 